\title{
Chemoembolization in Patients with Hepatocellular Carcinoma
}

\author{
Riccardo Lencioni \\ Division of Diagnostic Imaging and Intervention, Pisa University School of Medicine, Pisa, Italy
}

\section{Key Words}

Chemoembolization · Hepatocellular carcinoma · Transcatheter therapy

\begin{abstract}
Transcatheter arterial chemoembolization (TACE) is the standard of care for nonsurgical patients with preserved liver function with large or multinodular noninvasive hepatocellular carcinoma (HCC) confined to the liver. The administration of an anticancer-in-oil emulsion followed by embolic agents is the most popular TACE technique; however, the introduction of embolic, drug-eluting beads (DEB) has provided an alternative to conventional regimens. Experimental studies have shown that DEB-TACE results in a safe pharmacokinetic profile and effective tumor killing in animal models. Clinical experiences have confirmed that DEBTACE provides a combined ischemic and cytotoxic effect locally, with significantly reduced drug-related toxicity and liver toxicity compared with conventional TACE. The addition of molecular targeted drugs to the therapeutic armamentarium for HCC has prompted the design of clinical trials aimed at investigating the synergies between TACE and systemic treatments. Combining TACE with antiangiogenic agents represents a promising strategy because TACE is thought to cause local hypoxia, resulting in a temporary increase in levels of vascular endothelial growth factor. Recently, a large phase 2, randomized, double-blind, placebocontrolled trial (the SPACE study) indicated that the concurrent administration of DEB-TACE and sorafenib has a manageable safety profile and suggested that the time to progression (TTP) and time to vascular invasion or extrahepatic spread may be improved compared with DEB-TACE alone. These data support the further evaluation of molecular targeted, systemically active agents in combination with DEB-TACE in a phase 3 setting.
\end{abstract}




\section{Introduction}

HCC is the sixth most common cancer and the third leading cause of cancer-related death worldwide [1]. Unlike the situation for most solid cancers, the future incidence and mortality rates for HCC are projected to increase in several regions around the world over the next 20 years, mostly as a result of the dissemination of hepatitis $C$ virus infection $[2,3]$. Despite the widespread implementation of surveillance programs of at-risk populations, the majority of patients with HCC are diagnosed late, when curative treatments cannot be applied [4]. In addition, in a high proportion of cases, the disease recurs after radical therapy $[5,6]$.

TACE is the current standard of care for patients presenting with multinodular HCC and relatively preserved liver function, with no cancer-related symptoms, and with no evidence of vascular invasion or extrahepatic spread, i.e., those classified as intermediate stage according to the Barcelona Clinic Liver Cancer (BCLC) staging system [7-9]. While conventional TACE comprising administration of an anticancer-in-oil emulsion followed by embolic agents is the most popular technique, the introduction of embolic, drug-eluting beads (DEB) has provided an attractive alternative to lipiodol-based regimens [10]. Experimental and clinical studies have shown that the use of DEB loaded with doxorubicin has a safe pharmacokinetic profile with lower systemic drug exposure and significantly reduced liver toxicity compared with conventional TACE [11-13].

The recent addition of molecular targeted drugs to the therapeutic armamentarium for HCC has prompted the design of clinical trials aimed at investigating the synergies between TACE and systemic treatments $[14,15]$. The combination of TACE with anti-angiogenic agents is appealing because the systemically active drug might curtail the post-TACE rise in levels of vascular endothelial growth factor (VEGF)-mediated signaling and at the same time target any tumor foci distant from the site of TACE. In this article, the current role of TACE in HCC management is reviewed, and early clinical experiences with the combination of TACE and molecular targeted therapies are discussed.

\section{Conventional TACE}

HCC exhibits intense neo-angiogenic activity during its progression. The rationale for TACE is that the intraarterial infusion of a drug such as doxorubicin or cisplatin with or without a viscous emulsion, followed by embolization of the blood vessel with gelatin sponge particles or other embolic agents, will result in a strong cytotoxic effect combined with ischemia [10]. The survival benefit of transarterial embolization (TAE) or TACE has been the subject of a few randomized controlled trials (RCTs), and these have provided inconsistent results [1622] (table 1). A cumulative meta-analysis of these studies, however, clearly indicated that the 2-year survival of patients with HCC not suitable for radical therapy who were treated with arterial embolization or chemoembolization is improved compared with conservative management or suboptimal therapies [23]. Sensitivity analysis showed a significant benefit of chemoembolization with cisplatin or doxorubicin by assessing 323 patients in four studies, but no benefit with embolization alone by assessing 215 patients in three studies [23].

In a recent Cochrane meta-analysis, the evidence supporting the benefits of TACE has been questioned [24]. The authors of the Cochrane review stated that, despite TACE being a part of current clinical practice, there is an absence of evidence of TACE or TAE having a beneficial effect on survival in participants with unresectable HCC [24]. However, several experts have questioned such a conclusion and have expressed concern over this Cochrane review $[25,26]$. It has been pointed out that the analysis included an RCT undertaken in patients with early HCC - in whom TAE (not TACE) was assessed in combination with local 


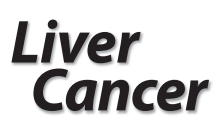

Table 1. RCTs comparing TACE or TAE with conservative management or suboptimal therapies for the treatment of HCC

\begin{tabular}{|c|c|c|c|c|c|}
\hline \multirow{2}{*}{ Study } & & \multirow{2}{*}{ No. of patients } & \multicolumn{3}{|c|}{ Overall survival (\%) } \\
\hline & & & 1 year & 2 year & $\mathrm{p}$ value \\
\hline \multirow[t]{3}{*}{ Lin et al. [16] } & TAE (Gelfoam + Ivalon) & 21 & 42 & 25 & NS \\
\hline & TAE + IV 5-fluorouracil & 21 & 20 & 20 & \\
\hline & IV 5-fluorouracil & 21 & 13 & 13 & \\
\hline \multirow[t]{2}{*}{ Pelletier et al. [17] } & TACE (doxorubicin, Gelfoam) & 21 & 24 & NA & NS \\
\hline & Conservative management & 21 & 33 & NA & \\
\hline \multirow[t]{2}{*}{$\mathrm{GETCH}^{*}[18]$} & TACE (cisplatin, Gelfoam) & 50 & 62 & 38 & NS \\
\hline & Conservative management) & 46 & 43 & 26 & \\
\hline \multirow[t]{2}{*}{ Bruix et al. [19] } & TAE (Gelfoam + coils) & 40 & 70 & 49 & NS \\
\hline & Conservative management & 40 & 72 & 50 & \\
\hline \multirow[t]{2}{*}{ Pelletier et al. [20] } & TACE (cisplatin, Gelfoam) + tamoxifen & 37 & 51 & 24 & NS \\
\hline & Tamoxifen & 36 & 55 & 26 & \\
\hline \multirow[t]{2}{*}{ Lo et al. [21] } & TACE (cisplatin, Gelfoam) & 40 & 57 & 31 & 0.002 \\
\hline & Conservative management & 39 & 32 & 11 & \\
\hline \multirow[t]{3}{*}{ Llovet et al. [22] } & TACE (doxorubicin, Gelfoam) & 40 & 82 & 63 & $0.009 * *$ \\
\hline & TAE (Gelfoam) & 37 & 75 & 50 & \\
\hline & Conservative management & 35 & 63 & 27 & \\
\hline
\end{tabular}

A meta-analysis of these trials identified a distinct survival benefit for TACE compared with the control [23]. IV = intravenous; NA = not available; NS = not significant. * Groupe d'Etude et de Traitement du Carcinome Hépatocellulaire. ${ }^{* *}$ Chemoembolization vs conservative management (TAE vs conservative management, NS; TACE vs TAE, NS).

ablation - and that it excluded two major trials that found improved survival because of risk of bias according to the Cochrane criteria $[25,26]$.

The outcome of TACE appears to be dependent on careful patient selection. In a RCT that recruited patients with compensated cirrhosis (70\% in Child-Pugh A), no cancer-related symptoms [81\% with an Eastern Cooperative Oncology Group (ECOG) performance status of $0]$, and large or multinodular HCC without vascular invasion or extrahepatic spread, 2-year survival after conventional TACE reached $63 \%$, compared with $27 \%$ for the untreated control arm $(p=0.009)$ [22]. In contrast, in another RCT, the use of broader enrollment criteria with inclusion of patients with symptoms or limited portal vein invasion resulted in a 2-year survival of only $31 \%$ [21]. This figure was still better than that of the untreated control group (2-year survival, $11 \% ; p=0.002$ ); however, no survival benefit was identified in subgroup analysis of patients presenting with portal vein invasion [21].

As a result of these investigations, TACE has become established as the standard of care for patients who meet the criteria for the intermediate stage of the BCLC staging system, i.e., those who are not suitable candidates for curative treatment options (surgical resection, liver transplantation, or image-guided ablation) but present with multinodular HCC and relatively preserved liver function and without cancer-related symptoms, vascular invasion, or extrahe- 
patic spread [7-9]. The tolerability of conventional TACE seems to be affected by the type of regimen and the frequency of the treatment. The Groupe d'Etude et de Traitement du Carcinome Hépatocellulaire evaluated a treatment schedule of conventional TACE with cisplatin every 2 months in patients with unresectable HCC but without severe liver disease [18]. This schedule was associated with frequent acute liver failure, with 30 of the 50 treated patients reporting this adverse event. Conversely, Llovet and colleagues evaluated a schedule of conventional TACE treatment with doxorubicin at baseline, 2 months, 6 months, and then every 6 months thereafter, with only 2 of 40 patients undergoing TACE eventually developing liver failure [22]. However, no RCTs have been designed to fully evaluate the optimum frequency of delivery [27]. There is also a lack of consensus regarding the use and type of chemotherapy agent or embolic material $[27,28]$.

\section{Drug-Eluting Bead TACE}

The ideal TACE scheme should allow maximum and sustained concentration of chemotherapeutic drug within the tumor with minimal systemic exposure combined with calibrated tumor vessel obstruction [10]. The recent introduction of embolic microspheres that have the ability to actively sequester doxorubicin hydrochloride from solution and release it in a controlled and sustained fashion has been shown to substantially diminish the amount of chemotherapy that reaches the systemic circulation compared with lipiodol-based regimens, thus increasing the local concentration of the drug and the antitumoral efficacy [11, 12].

In a multicenter phase 2 RCT including 201 European patients (PRECISION V), use of doxorubicin-eluting beads resulted in a marked reduction in liver toxicity and drug-related adverse events compared with conventional TACE with doxorubicin $[12,13]$. The mean maximum aspartate aminotransferase increase and alanine aminotransferase increase in the DEB bead group were $50 \%$ and $41 \%$ lower than those in the conventional TACE group $(\mathrm{p}<0.001)$. Because of the improved safety and tolerability profile, high-dose doxorubicin treatment could be applied according to the planned schedule in all members of the DEB group, resulting in consistently high rates of objective response and disease control in all subgroup analyses. In contrast to the results for the DEB arm, the objective response rate (ORR) and the disease control rate (DCR) for conventional TACE in the subgroups of patients with more advanced disease were significantly reduced $(p=0.038$ for ORR; $p=0.026$ for DCR), although the differences were not statistically significant in the overall patient population (ORR, $52 \%$ vs $44 \%$; DCR, $63 \%$ vs $52 \%$ ). A recent case-control study conducted in Asian patients with HCC confirmed higher ORR for DEB-TACE compared with conventional TACE [29] (table 2).

The added value of microspheres loaded with a chemotherapeutic agent over bland embolic microspheres was demonstrated by a RCT comparing beads loaded with doxorubicin versus bland embolization performed with embolic microspheres with similar characteristics. The rate of tumor progression at 12 months was significantly lower in the DEB arm than in the bland embolization arm ( $46 \%$ vs $78 \%, p=0.002)$, and the TTP increased from $36.2 \pm$ 9.0 weeks to $42.4 \pm 9.5$ weeks ( $p=0.008$ ) [30]. Another investigation assessed the degree of necrosis in explanted livers after chemoembolization with epirubicin-loaded DEB versus bland embolization in patients on a transplant waiting list: DEB-TACE achieved complete necrosis in $77 \%$ of lesions, whereas bland embolization achieved complete necrosis in only $27 \%$ of lesions ( $p=0.043$ ) [31].

Despite the absence of phase 3 trials comparing DEB-TACE versus conventional regi- 


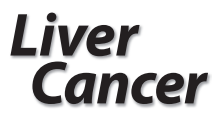

Table 2. Selected phase 2 trials comparing conventional TACE with DEB-TACE for the treatment of HCC

\begin{tabular}{|c|c|c|c|c|c|}
\hline Study/Subgroup & Design & Treatment group & No. of patients & ORR & $\mathrm{p}$ value \\
\hline \multirow[t]{4}{*}{ Lammer et al. [12] } & $\mathrm{RCT}^{*}$ & & & & NS \\
\hline & & cTACE & 108 & 44 & \\
\hline & & DEB-TACE & 93 & 52 & \\
\hline & & & & & 0.038 \\
\hline \multirow{2}{*}{ Child-Pugh B } & & cTACE & 19 & 21 & \\
\hline & & DEB-TACE & 16 & 44 & \\
\hline \multirow{2}{*}{ ECOG 1} & & CTACE & 28 & 29 & \\
\hline & & DEB-TACE & 19 & 63 & \\
\hline \multirow{2}{*}{ Bilobar disease } & & cTACE & 45 & 40 & \\
\hline & & DEB-TACE & 41 & 49 & \\
\hline \multirow{2}{*}{ Recurrent disease } & & cTACE & 13 & 31 & \\
\hline & & DEB-TACE & 11 & 55 & \\
\hline \multirow[t]{3}{*}{ Song et al. [29] } & Case-control study ** & & & & 0.001 \\
\hline & & cTACE & 20 & 30 & \\
\hline & & DEB-TACE & 20 & 85 & \\
\hline
\end{tabular}

cTACE = conventional transarterial chemoembolization; DEB-TACE = drug-eluting bead transcatheter arterial chemoembolization. * Primary endpoint: 6-month ORR; ** Primary endpoint: 1-month ORR.

mens, DEB-TACE has been increasingly used as the first-line transcatheter treatment for HCC [32]. DEB-TACE provides levels of consistency and repeatability not available with conventional TACE; it also offers the opportunity to implement a standardized approach to HCC treatment [32]. In a recent report, the median survival of 48 months in a cohort of 104 European patients with HCC treated with DEB-TACE appears to be far superior than that reported for conventional TACE [33].

\section{Response Assessment}

The assessment of tumor response is of the utmost importance in patients undergoing TACE [28]. Unfortunately, conventional methods for response assessment, such as the Response Evaluation Criteria In Solid Tumors (RECIST), have limited predictive value in HCC patients treated with TACE $[34,35]$. These criteria rely on tumor shrinkage alone as a measure of antitumor activity, an assumption that is valid only when cytotoxic drugs are used. TACE induces direct tumor necrosis, and its anticancer efficacy is not paralleled by a reduction in overall tumor load, but rather by a reduction in viable tumor, as recognized by contrastenhanced radiologic imaging [35]. A modification of the RECIST criteria - named modified RECIST (mRECIST) for HCC - was first proposed by a panel of experts in 2008 [7], and then was further expanded in 2010 [36]. The basis of this proposal is that the diameter of target lesions with viable tumor should guide all measurements. In addition, specific modifications of the original criteria have been introduced regarding assessment of vascular invasion, lymph nodes, ascites, pleural effusion, and new lesions [36].

Tumor response measured by mRECIST after TACE or systemic therapy has been shown to be associated with survival [37-39]. In a European investigation, tumor response was determined using conventional RECIST, European Association for the Study of the Liver (EASL), 
Table 3. Tumor response assessment in HCC patients who underwent TACE

\begin{tabular}{lcc}
\hline Response & $\begin{array}{c}\text { EASL } \\
\text { Overall survival HR (95\% CI) }\end{array}$ & $\begin{array}{c}\text { mRECIST } \\
\text { Overall survival HR (95\% CI) }\end{array}$ \\
Complete response & 1.0 & 1.0 \\
Partial response & $2.99(2.14-4.17)^{*}$ & $2.75(1.96-3.87)^{*}$ \\
Stable disease & $3.49(1.71-7.10)^{*}$ & $6.32(3.67-10.90)^{*}$ \\
Progressive disease & $15.63(9.51-25.69)$ & $16.06(9.76-26.43)$ \\
\hline
\end{tabular}

Both EASL and mRECIST criteria were independent predictors of overall survival, but only the mRECIST model yielded a significant difference in the probability of survival across all different response categories [39]. mRECIST = Modified Response Evaluation Criteria In Solid Tumors. ${ }^{*}$ Partial response vs stable disease: $\mathrm{p}=0.71$ (not significant) for EASL and $\mathrm{p}=0.002$ for $\mathrm{mRECIST}$.

and mRECIST criteria in 83 patients with HCC who underwent transarterial (chemo) embolization [38]. No significant difference was found between responders and nonresponders according to conventional RECIST. In contrast, the difference between responders and nonresponders reached statistical significance for both the EASL and mRECIST assessments. Patients who showed objective response according to mRECIST $(n=48)$ had a median overall survival of 20.7 months versus 13.3 months for nonresponders $(n=35)$. The association between overall survival and mRECIST responses was retained in a multivariate analysis [38].

These findings were confirmed by an Asian study of 332 patients with intermediate stage HCC and Child-Pugh A cirrhosis who underwent serial TACE [39]. All measurable target lesions of $1 \mathrm{~cm}$ or larger in diameter were measured according to conventional RECIST, EASL, and mRECIST. The Cox regression model revealed that - in contrast to conventional RECIST - both EASL and mRECIST criteria were independent predictors of overall survival $(\mathrm{p}<0.001)$. In addition, mRECIST yielded a significant difference in the probability of survival across all different response categories ( $p<0.001$ for each comparison). However, there was no significant difference in the probability of survival between partial response and stable disease categories with the EASL criteria ( $p=0.71$ ) [39] (table 3).

The recent Clinical Practice Guidelines jointly issued by EASL and the European Organization for Research and Treatment of Cancer (EASL-EORTC guidelines) state that assessment of response in HCC should be based on mRECIST criteria by performing contrast-enhanced computed tomography or magnetic resonance imaging 1 month after initial treatment and at least every 3 months during follow-up [9].

\section{Synergies and Combination Strategies}

An important limitation of all TACE regimens is the high rate of tumor recurrence. In RCTs, a sustained response lasting more than 3-6 months was observed in only $28-35 \%$ of patients receiving conventional TACE, and in nonresponders, no survival benefit was identified compared with best supportive care [21,22]. Even in those patients in whom an initial response was achieved, the 3-year cumulative rate of intrahepatic recurrence reached $65 \%$, with recurrent tumor showing significantly shorter median doubling time [40]. As a result, the 3-year survival rate of TACE-treated patients did not exceed 26-29\% [21, 22].

Increased understanding of the molecular signaling pathways involved in HCC has led 
to the development of molecular targeted therapies aimed at inhibiting tumor cell proliferation and angiogenesis. Sorafenib, a multi-kinase inhibitor with antiangiogenic and antiproliferative properties, has been shown to prolong median overall survival and median time to radiological progression compared with a placebo in RCTs and has become the current standard of care for patients with advanced-stage tumors not suitable for surgical or loco-regional therapies $[41,42]$.

Tumor recurrence following TACE is characterized by increased VEGF production and subsequent angiogenesis. Moreover, TACE increases VEGF expression in the residual surviving cancerous tissue [43] and induces expression of other pro-angiogenic factors, such as hypoxia-inducible factor 1 alpha [44]. Based on these findings, the combination of TACE with anti-angiogenic agents would appear to be a rational approach.

DEB ensures minimal systemic exposure to the chemotherapeutic agent at the time of TACE and is appealing for combination regimens based on mechanisms that are theoretically synergistic. DEB-TACE has been shown to be safe and effective, with significantly reduced systemic drug exposure compared with conventional TACE regimens. Sorafenib has demonstrated efficacy and safety in patients with advanced HCC, with activity against both tumor cells and endothelial cells. In a prospective, single-center phase 2 study conducted at the Johns Hopkins University School of Medicine, the safety and responses of a combined protocol involving sorafenib $400 \mathrm{mg}$ twice per day and DEB-TACE were assessed in 35 patients [45]. Although most patients experienced at least one grade 3-4 toxicity, most toxicities were minor (grade 1-2, 83\% vs grade 3-4,17\%), and preliminary efficacy data were promising.

The phase 2, randomized, double-blind, placebo-controlled SPACE study (Sorafenib or Placebo in Combination with DEB-TACE for Intermediate-Stage HCC) was the first global trial on the use of TACE in the treatment of HCC [46]. The objective of the study was to evaluate the efficacy and safety of sorafenib in combination with DEB-TACE in patients with intermediatestage HCC. The study was conducted at 85 sites across Europe, North America, and the AsiaPacific region. Patients were eligible if they had asymptomatic, unresectable, multinodular tumors without vascular invasion or extrahepatic spread (EHS); Child-Pugh A liver functional status; and ECOG performance status 0 [46]. Patients were randomized to receive sorafenib $400 \mathrm{mg}$ b.i.d. or matching placebo continuously (1 cycle was 4 weeks) until progression. All patients underwent DEB-TACE (150 mg doxorubicin) 3-7 days after receiving the first dose of the study drug, and then on day 1 ( \pm 4 days) of cycles 3,7 , and 13 , and every 6 cycles thereafter. Patients were allowed optional DEB-TACE sessions between cycles 7 and 13 and cycles 13 and 19 if deemed necessary by the investigator. The primary endpoint was TTP by independent review (predefined alpha $=0.15$ ). Secondary endpoints were overall survival, time to vascular invasion or extrahepatic spread, time to untreatable progression (TTUP), and safety [46].

Of 452 patients screened, 307 were randomized to sorafenib $(n=154)$ or placebo $(n=153)$. The hazard ratio (HR) for TTP was 0.797 [95\% confidence interval (CI), 0.588-1.080; $\mathrm{p}=$ 0.072]. Median TTP (50th percentile) was 169 days and 166 days in the sorafenib and placebo groups, respectively; TTPs at the 25th/75th percentiles (preplanned) were 112/285 days in the sorafenib group and 88/224 days and placebo group. There were no unexpected safety findings. Median treatment durations in the sorafenib and placebo groups were 4.8 and 6.3 months, respectively, and the median daily dose of study drug was $566 \mathrm{mg}$ and $791 \mathrm{mg}$, respectively [46]. Table 4 gives a summary of the efficacy data.

The findings of the SPACE study were supported by a recently published prospective, single-arm, phase 2 study including 50 Asian patients with unresectable HCC (82\% in BCLC stage $\mathrm{B}$ and $18 \%$ in BCLC stage C) [47]. In this study, concurrent treatment with TACE and sorafenib showed promising efficacy results (median TTP, 7.1 months; $95 \%$ CI, 4.8-7.5 months) and a manageable safety profile with a median dose intensity of sorafenib of $68.7 \%$ (range, 37.3$100 \%$ ) of $800 \mathrm{mg}$ daily [47]. Nevertheless, the encouraging signal indicated by the SPACE trial 
Table 4. Phase 2, randomized, double-blind, placebo-controlled SPACE study (Sorafenib or Placebo in Combination with DEB-TACE for Intermediate-Stage HCC)

\begin{tabular}{lcccc}
\hline Assessment* & TTP & $\begin{array}{c}\text { Overall } \\
\text { survival }\end{array}$ & Time to VI/EHS & TTUP \\
\hline HR & 0.797 & 0.898 & 0.621 & 1.586 \\
$95 \%$ CI & $0.588-1.080$ & $0.606-1.330$ & $0.321-1.200$ & $1.200-2.096$ \\
p value (1-sided) $^{\dagger}$ & 0.072 & 0.295 & 0.076 & 0.999 \\
\hline
\end{tabular}

The combination of DEB-TACE and sorafenib prolonged TTP (primary endpoint) and time to vascular invasion or extrahepatic spread compared with DEB-TACE alone $(\mathrm{p}=0.072$ and $\mathrm{p}=0.076$, respectively; predefinedalpha $=0.15$ ] [46].VI=vascularinvasion.*intention-to-treatpopulation(allpatientsrandomized); ${ }^{\dagger}$ predefined alpha $=0.15 ; \S$ median was not reached in either group.

requires confirmation with data from ongoing phase 3 trials.

Several questions remain as we attempt to improve treatment outcomes in HCC patients. The pathophysiologic complexity of HCC, balanced with a goal of providing effective tumor therapy with preservation of organ function, makes optimal treatment choice clinically challenging [48]. An understanding of which features of HCC and patient health may predict the clinical outcome of combination regimens is essential for determining individualized, evidence-based therapeutic strategies.

\section{References}

1 Parkin DM, Bray F, Ferlay J, Pisani P: Global cancer statistics, 2002. CA Cancer J Clin 2005;55:74-108.

2 Olsen AH, Parkin DM, Sasieni P: Cancer mortality in the United Kingdom: projections to the year 2025. Br J Cancer 2008;99:1549-1554.

3 Davis GL, Alter MJ, El-Serag H, Poynard T, Jennings LW: Aging of the hepatitis C virus (HCV)-infected persons in the United States: A multiple cohort model of HCV prevalence and disease progression. Gastroenterology 2010;138:513-521.

4 Kim WR, Gores GJ, Benson JT, Therneau TM, Melton LJ 3rd: Mortality and hospital utilization for hepatocellular carcinoma in the United States. Gastroenterology 2005;129:486-493.

5 Poon RT, Fan ST, Ng IO, Lo CM, Liu CL, Wong J: Different risk factors and prognosis for early and late intrahepatic recurrence after resection of hepatocellular carcinoma. Cancer 2000;89:500-507.

6 Cucchetti A, Piscaglia F, Caturelli E, et al: Comparison of recurrence of hepatocellular carcinoma after resection in patients with cirrhosis to its occurrence in a surveilled cirrhotic population. Ann Surg Oncol 2009;16:413-422.

7 Llovet JM, Di Bisceglie AM, Bruix J, et al: Panel of Experts in HCC-Design Clinical Trials: Design and endpoints of clinical trials in hepatocellular carcinoma. J Natl Cancer Inst 2008;100:698-711.

8 Bruix J, Sherman M: Management of hepatocellular carcinoma: an update. Hepatology 2011;53:10201022.

9 EASL-EORTC Clinical Practice Guidelines: Management of hepatocellular carcinoma. European Association for the Study of the Liver; The European Organization for Research and Treatment of Cancer. J Hepatol. 2012;56:908-943.

10 LencioniR: Loco-regional treatment of hepatocellular carcinoma. Hepatology 2010;52:762-773.

11 Varela M, Real MI, Burrel M, et al: Chemoembolization of hepatocellular carcinoma with drug eluting beads: efficacy and doxorubicin pharmacokinetics. J Hepatol 2007;46:474-481.

12 Lammer J, Malagari K, Vogl T, et al: Prospective randomised study of doxorubicin-eluting-bead embolization in the treatment of hepatocellular carcinoma: results of the PRECISION V study. Cardiovasc Intervent Radiol 2010;33:41-52.

13 Vogl TJ, Lammer J, Lencioni R, Malagari K, Watkinson A, Pilleul F, Denys A, Lee C: Liver, gastrointestinal, and cardiac toxicity in intermediate hepatocellular carcinoma treated with PRECISION TACE with drugeluting beads: results from the PRECISION V randomized trial. AJR Am J Roentgenol 2011;197:W562-570.

14 Lencioni R: Management of hepatocellular carcinoma with transarterial chemoembolization in the era of systemic targeted therapy. Crit Rev Oncol Hematol 2012 [Epub ahead of print].

15 Lencioni R, Crocetti L: Loco-regional treatment of hepatocellular carcinoma. Radiology 2012;262:43-58.

16 Lin DY, Liaw YF, Lee TY, Lai CM: Hepatic arterial embolization in patients with unresectable hepatocellular carcinoma - a randomized controlled trial. Gastroenterology 1988;94:453-456. 
17 Pelletier G, Roche A, Ink 0, et al: A randomized trial of hepatic arterial chemoembolization in patients with unresectable hepatocellular carcinoma. J Hepatol 1990;11:181-184.

18 A comparison of lipiodol chemoembolization and conservative treatment for unresectable hepatocellular carcinoma. Groupe d'Etude et de Traitement du Carcinome Hépatocellulaire.i N Engl J Med 1995;332:12561261.

19 Bruix J, Llovet JM, Castells A, et al: Transarterial embolization versus symptomatic treatment in patients with advanced hepatocellular carcinoma: results of a randomized, controlled trial in a single institution. Hepatology 1998;27:1578-1583.

20 Pelletier G, Ducreux M, Gay F, et al: Treatment of unresectable hepatocellular carcinoma with lipiodol chemoembolization: a multicenter randomized trial. J Hepatol 1998;29:129-134.

21 Lo CM, Ngan H, Tso WK, et al: Randomized controlled trial of transarterial lipiodol chemoembolization for unresectable hepatocellular carcinoma. Hepatology 2002;35:1164-1171.

22 Llovet JM, Real MI, Montana X, et al: Arterial embolisation or chemoembolisation versus symptomatic treatment in patients with unresectable hepatocellular carcinoma: a randomised controlled trial. Lancet 2002;359:1734-1739.

23 Llovet JM, Bruix J: Systematic review of randomized trials for unresectable hepatocellular carcinoma: Chemoembolization improves survival. Hepatology 2003;37:429-442.

24 Oliveri RS, Wetterslev J, Gluud C: Transarterial (chemo) embolisation for unresectable hepatocellular carcinoma. Cochrane Database Syst Rev 2011;3:CD004787.

25 Ray CE, Haskal ZJ, Geschwind JF, Funaki BS: The use of transarterial chemoembolization in the treatment of unresectable hepatocellular carcinoma: A response to the Cochrane Collaboration Review of 2011. J Vasc Interv Radiol 2011;22:1693-1696.

26 Forner A, Llovet JM, Bruix J: Chemoembolization for intermediate HCC: Is there proof of survival benefit? J Hepatol 2011 [Epub ahead of print].

27 Giannini EG, Bodini G, Corbo M, et al: Impact of evidence-based medicine on treatment of patients with unresectable hepatocellular carcinoma. Aliment Pharmacol Ther 2010;31:493-501.

28 Raoul JL, Sangro B, Forner A, et al: Evolving strategies for the management of intermediate-stage hepatocellular carcinoma: available evidence and expert opinion on the use of transarterial chemoembolization. Cancer Treat Rev 2011;37:212-220.

29 Song MJ, Park CH, Kim JD, et al: Drug-eluting bead loaded with doxorubicin versus conventional Lipiodolbased transarterial chemoembolization in the treatment of hepatocellular carcinoma: a case-control study of Asian patients. Eur J Gastroenterol Hepatol 2011;23:521-527.

30 Malagari K, Pomoni M, Kelekis A, et al: Prospective randomized comparison of chemoembolization with doxorubicin-eluting beads and bland embolization with Beadblock for hepatocellular carcinoma. Cardiovasc Intervent Radiol 2010;33:541-551.

31 Nicolini A, Martinetti L, Crespi S, Maggioni M, Sangiovanni A: Transarterial chemoembolization with epirubicin-eluting beads versus transarterial embolization before liver transplantation for hepatocellular carcinoma. J Vasc Interv Radiol 2010;21:327-332.

32 Lencioni R, de Baere T, Burrel M, et al: Transcatheter treatment of hepatocellular carcinoma with doxorubicin-loaded DC Bead (DEBDOX): technical recommendations. Cardiovasc Intervent Radiol 2012 [Epub ahead of print].

33 Burrel M, Reig M, Forner A, et al: Survival of patients with hepatocellular carcinoma treated by transarterial chemoembolization (TACE) using DC Beads. Implications for clinical practice and trial design. J Hepatol 2012 [Epub ahead of print].

34 Therasse P, Arbuck SG, Eisenhauer EA, et al: New guidelines to evaluate the response to treatment in solid tumors. J Natl Cancer Inst 2000;92:205-216.

35 Forner A, Ayuso C, Varela M, et al: Evaluation of tumor response after locoregional therapies in hepatocellular carcinoma: are response evaluation criteria in solid tumors reliable? Cancer 2009;115:616-623.

36 Lencioni R, Llovet JM: Modified RECIST (mRECIST) assessment for hepatocellular carcinoma. Semin Liver Dis 2010;30:52-60.

37 Edeline J, Boucher E, Rolland Y, et al: Comparison of tumor response by response evaluation criteria in solid tumors (RECIST) and modified RECIST in patients treated with sorafenib for hepatocellular carcinoma. Cancer 2012 [Epub ahead of print].

38 Gillmore R, Stuart S, Kirkwood A, et al: EASL and mRECIST responses are independent prognostic factors for survival in hepatocellular cancer patients treated with transarterial embolisation. J Hepatol 2012 [Epub ahead of print].

39 Shim JH, Lee HC, Kim SO, et al: Which response criteria best help predict survival of patients with hepatocellular carcinoma following chemoembolization? A validation study of old and new models. Radiology 2012;262:708-718.

40 Woo HY, Jang JW, Choi JY, et al: Tumor doubling time after initial response to transarterial chemoembolization in patients with hepatocellular carcinoma. Scand J Gastroenterol 2010;45:332-339.

41 Llovet JM, Ricci S, Mazzaferro V, et al: Sorafenib in advanced hepatocellular carcinoma. N Engl J Med 2008;359:378-390.

42 Cheng AL, Kang YK, Chen Z, et al: Efficacy and safety of sorafenib in patients in the Asia-Pacific region with advanced hepatocellular carcinoma: a phase III randomised, double-blind, placebo-controlled trial. Lancet Oncol 2009;10:25-34.

43 Wang B, Xu H, Gao ZQ, Ning HF, Sun YQ, Cao GW: Increased expression of vascular endothelial growth factor in hepatocellular carcinoma after transcatheter arterial chemoembolization. Acta Radiol 2008;49:523- 
529.

44 Virmani S, Rhee TK, Ryu RK, et al: Comparison of hypoxia-inducible factor-1alpha expression before and after transcatheter arterial embolization in rabbit VX2 liver tumors. J Vasc Interv Radiol 2008;19:14831489.

45 Pawlik TM, Reyes DK, Cosgrove D, Kamel IR, Bhagat N, Geschwind J: Phase II trial of sorafenib combined with concurrent transarterial chemoembolization with drug-eluting beads for hepatocellular carcinoma. J Clin Oncol 2011;29:3960-3967.

46 Lencioni R, Llovet JM, Han G, et al: Sorafenib or placebo in combination with transarterial chemoembolization (TACE) with doxorubicin-eluting beads (DEBDOX) for intermediate-stage hepatocellular carcinoma (HCC): Phase II, randomized, double-blind SPACE trial. J Clin Oncol 2012;30 (suppl 4; abstr LBA154).

47 Park JW, Koh YH, Kim HB, et al: Phase II study of concurrent transarterial chemoembolization and sorafenib in patients with unresectable hepatocellular carcinoma.J Hepatol 2012 [Epub ahead of print].

48 Gish RG, Lencioni R, Di Bisceglie AM, Raoul JL, Mazzaferro V: Role of the multidisciplinary team in the diagnosis and treatment of hepatocellular carcinoma. Expert Rev Gastroenterol Hepatol 2012;6:173-185. 